\title{
Fatores determinantes da dependência de crack na tríplice fronteira - Brasil, Bolívia e Paraguai
}

\author{
Determinants of crack addiction in the tri-border - Brazil, Bolivia and Paraguay \\ Déterminants de la dépendance à crack dans la triple frontière - Brésil, Bolivie et \\ Paraguay
}

Factores determinantes de la adicción al crack en la triple frontera - Brasil, Bolivia y Paraguay

\author{
André Barciela Veras* \\ (barcielaveras@hotmail.com) \\ Recebido em 07/11/2014, revisado e aprovado em 07/07/2015; aceito em 20/08/2015 \\ DOI: http://dx.doi.org/10.20435/1518-70122016112
}

\begin{abstract}
Resumo: Através da experiência dos profissionais da assistência, docentes e usuários, caracterizamos os fatores geradores da dependência de crack/pasta-base na região, utilizando a Matriz de Corvalán. O uso da Matriz de Corvalán para uma condição de saúde diferente das doenças infecto-parasitárias mostrou-se produtivo. Nesse caso em particular, para a ampliação do entendimento dos determinantes ambientais para a dependência de crack.

Palavras-chave: Crack. Matriz de Corvalán. Saúde Ambiental.

Abstract: Using the experience of assistance professionals, professors and health users, the environmental reasons for the occurrence of crack dependence in the state of MS were characterized through the Matrix of Corvalán. The use of Matrix of Corvalán seemed productive for a condition different of the infectious-parasites diseases. In this particular case, helping in broader understandings of environmental determinants of crack addiction.

Key words: Crack. Matrix of Corvalán. Environmental Health.

Résumé: Grâce à l'expérience de l'assistance professionnelle, les enseignants et les utilisateurs, nous caractérisons les facteurs générateurs de la dépendance de le crack / dossier-base dans la région, en utilisant la matrice Corvalan. Utilisation de la matrice Corvalan pour une condition différente de maladies infectieuses et parasitaires santé avérée productive. Dans ce cas particulier, d'élargir la compréhension des déterminants environnementaux pour dépendance au crack.

Mots-clés: Crack, Matrix Corvalan, la santé environnementale

Resumen: A través de la experiencia de los profesionales de la atención, los maestros y los usuarios, se caracterizan los factores que generan la dependencia del crack de la región, utilizando la Matriz de Corvalán. El uso de la matriz Corvalán para una condición de salud diferente de las enfermedades infecciosas y parasitarias resultó ser productivo. En este caso particular, para ampliar la comprensión de los determinantes ambientales para la adicción al crack.

Palabras clave: Crack. Matrix Corvalan. Saúde Ambiental.
\end{abstract}

\section{INTRODUÇÃO}

A dependência de crack vem se caracterizando como um problema de saúde pública no Brasil, nem tanto pela prevalência do uso (NAPPO et al., 2012), e sim pela intensidade da dependência e pelos danos para a saúde dela resultantes (DUALIBI et al., 2008). Em virtude desses aspectos, a dependência de Crack se tornou uma das importantes demandas em ambientes ambulatoriais e hospitalares de tratamento (PASSOS; CAMACHO, 1998; PEIXOTO et al., 2010). O primeiro estudo nacional de prevalência identificou, em 2001 (CARLINI et al., 2002), uma frequência de uso do crack ao longo da vida de $0,6 \%$, aumentando para $0,7 \%$ no segundo estudo em 2005 (CARLINI et al., 2007). Na região Centro-Oeste, a frequência do uso ao longo da vida foi de 0,3\% em 2005 e, neste último levantamento, Mato Grosso do Sul (MS) foi pesquisado apenas em sua capital, Campo Grande.

O Estado do MS está situado em uma região de tríplice fronteira formada pelo Brasil, Bolívia e Paraguai. O posicionamento na região de fronteira de alguns municípios, em especial Corumbá (103.703 habitantes) e Ponta Porã (77.872 hab.) (IBGE, 2010), produz um constante trânsito internacional de pessoas pela existência de cidades justapostas na Bolívia (Puerto Quijarro \& Puerto Suarez-Corumbá) e no Paraguai (Pedro Juan Caballero-Ponta Porã). Estas últimas duas

\footnotetext{
* Universidade Católica Dom Bosco (UCDB), Campo Grande, Mato Grosso do Sul, Brasil.
} 
cidades formam uma área de conurbação, inexistindo um controle possível dos limites entre Paraguai e Brasil. Entre os dois municípios, passou a existir uma "linha" de imprecisão de jurisdição, onde ocorre uma prática não reprimida de tráfico, consumo de drogas e prostituição na rua.

Sendo a Bolívia e o Paraguai importantes produtores/exportadores de drogas (BRASIL, 2012), o MS se tornou uma das rotas de passagem do tráfico, assim como um mercado que se beneficia do baixo custo pela proximidade de áreas de produção. Segundo o Sistema Nacional de Dados Estatísticos de Repressão a Entorpecentes, entre os anos de 2008 e 2012, a Polícia Federal apreendeu $292,4 \mathrm{Kg}$ de crack / pasta-base no MS, o que corresponde a apenas 3\% das apreensões nacionais.

O Brasil vem apresentando significativo crescimento econômico e desenvolvimento social e é um dos atores centrais da Declaração do Rio (Eco 92) e da produção da Agenda 21. Além disso, no ano de 2012, foi realizada, no Rio de Janeiro, a Rio+20. Diante de tais eventos, o desenvolvimento sustentável deve ser um conceito norteador das políticas nacionais. Dentro desse conceito, a saúde do Homem é um elemento central (UNITED NATIONS, 1993).

Múltiplos fatores ambientais afetam a saúde da população, formando complexas relações de causa e efeito. Para a exploração dessas relações de forma sistematizada, foi desenvolvida a estratégia da Matriz de Corvalán (CORVALÁN et al., 1999) ou “DPSEEA (Driving force - Pressure - State - Exposure - Effect - Action) framework" (WHO, 1999), nome adotado pela Organização Mundial de Saúde. Corvalán et al. (1999) estratificaram os fatores que afetam a saúde do Homem em quatro níveis: Força Motriz; Pressão; Estado; Exposição. Tais fatores se relacionam da seguinte forma: Uma Força Motriz é gerada por um fenômeno macrossocial ou político, que provoca uma Pressão sobre a sociedade. Tal pressão a modifica e movimenta, causando um novo Estado no ambiente. Quando o Homem se expõe a essa modificação no ambiente, ocorre um efeito potencial sobre a saúde dos indivíduos (Figura 1).
Através da experiência dos profissionais, docentes e usuários, procuramos caracterizar os fatores geradores da dependência de crack/pasta-base no MS utilizando como instrumento ordenador a Matriz de Corvalán. Esse instrumento foi utilizado pela primeira vez na literatura para caracterizar os determinantes ambientais causadores de um transtorno mental.

\section{METODOLOGIA}

Foi realizada uma oficina no principal evento regional de saúde mental do MS no ano de 2012 - III Encontro de CAPS de Campo Grande e V Jornada de Saúde Mental. O evento reúne profissionais da rede de saúde mental de múltiplas localidades do MS. A oficina contou com 25 participantes, entre estes, profissionais da rede de assistência familiar de usuário da saúde e docentes. Os participantes procuraram a oficina espontaneamente, quando foram apresentados ao instrumento (Matriz de Corvalán ou DPSEEA framework) pelo docente responsável e, posteriormente, divididos em 5 grupos de trabalho, cada um com cinco participantes.

Os grupos foram orientados a construir uma matriz. Em seguida, cada grupo apresentou sua matriz para todos os integrantes da oficina e uma matriz única, simultaneamente às apresentações, foi construída pelo autor deste artigo com o uso do programa Word, que estava sendo projetado por data-show para a adequada visualização por todos os presentes. Todos os dados apresentados pelos grupos foram inseridos na matriz final, sendo excluídos apenas os dados que repetiam determinantes já citados pelos grupos anteriores.

A atividade não precisou ser registrada em vídeo, visto que a matriz final foi construída no momento da oficina e, ao término desta, corrigida e aprovada pelos participantes. A oficina foi realizada em apenas um período e teve duração de 3 horas. Tais participantes representavam diferentes setores prestadores de atenção aos usuários de drogas do MS. As instituições e segmentos de atuação dos indivíduos presentes encontram-se resumidos na Tabela 1. 
Tabela 1 - Caracterização dos participantes envolvidos na construção da Matriz de Corvalan sobre a dependência de Crack.

\begin{tabular}{ll}
$\mathbf{N}$ & \multicolumn{1}{c}{ Vínculo Institucional } \\
\hline 8 & CAPS-ad de CG \\
4 & Hospital Psiquiátrico \\
3 & Comunidade terapêutica \\
2 & Hospital Geral \\
2 & Centro de Atenção Primária de CG \\
2 & Universidade \\
1 & CAPS-ad Corumbá \\
1 & Centro de Atenção ao Índio \\
1 & Familiar de usuário (CAPS-ad de CG) \\
1 & Assistência Social de CG \\
\hline
\end{tabular}

Total $=25$

CAPS-ad = Centro de Atenção Psicossocial; $\mathrm{CG}=$ Campo Grande, MS.

Foi utilizada a Matriz de Corvalán (CORVALÁN et al., 1999; WHO, 1999) como instrumento de sistematização do "brain storm" gerado pelos participantes da oficina. Tal ferramenta foi desenvolvida para uma identificação e sistematização iniciais dos fatores que geram um efeito sobre a saúde da população, efeito este revelado através de marcadores epidemiológicos.

No presente estudo, o efeito escolhido foi a Dependência de Crack / Pasta-base. A Matriz de Corvalán permite que a prevalência de uma determinada condição patológica não seja vista apenas como causada pela simples exposição do Homem a um contaminante presente no ambiente. A ferramenta procura contemplar todos os fatores que provocam a presença da ameaça ambiental, apresentando relações a serem analisadas por estudos qualitativos, que permitirão ações preventivas mais efetivas no âmbito da saúde pública e mais abrangentes do que a simples interrupção do contato entre o indivíduo e a ameaça no ambiente.

Como já referido, a Matriz de Corvalán estratifica os fatores que afetam a saúde do Homem em quatro níveis: Força Motriz; Pressão; Estado; Exposição; Efeito.

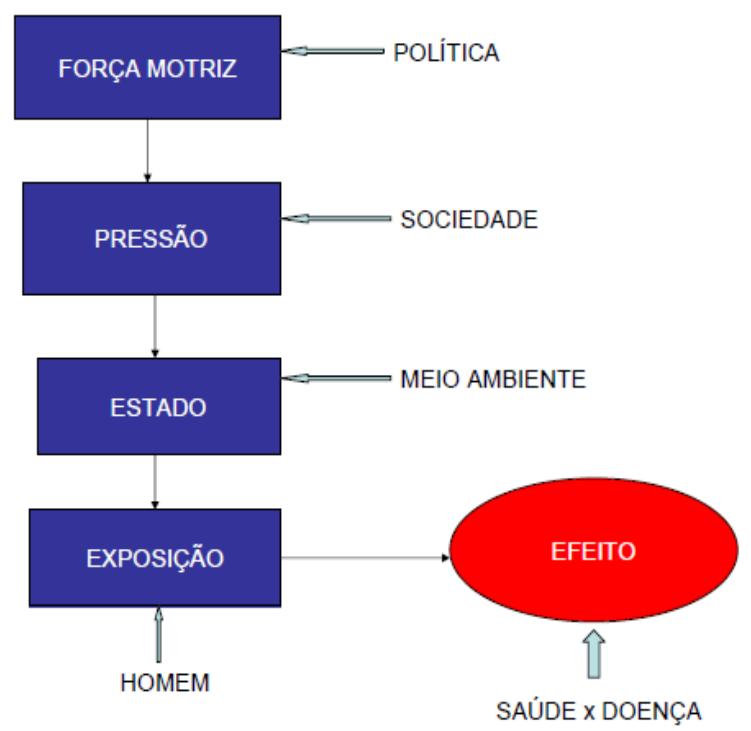

Figura 1 - Estratificação de efeitos para a ocorrência de uma afetação na saúde dos indivíduos, segundo Corvalán et al. (1999).

A matriz fornecida para os grupos participantes da oficina continha apenas o Efeito de "Dependência de Crack / Pasta-base". Sendo assim, cada grupo inseriu as próprias Forças Motrizes, Pressões, Estados e Exposições, além das inter-relações entre esses aspectos que achava cabível, a partir da experiência de trabalho de cada componente.

\section{RESULTADOS}

Através de uma oficina com profissionais de formação diversificada e provindos de múltiplos setores da sociedade (Tabela 1) foi construída uma Matriz de Corvalán a partir do Efeito de "Dependência de Crack / Pasta-base" (Figura 2).

Seis Forças Motrizes foram apontadas como contribuintes para a ocorrência de dez diferentes Pressões sobre o ambiente, criando onze Situações sociais que, por sua vez, geram outras onze Exposições favorecedoras para a dependência. Foram identificadas forças motrizes das esferas da Economia-Produtividade-Agricultura e das Políticas Educacional, de Saúde, Cultural e de Segurança Pública. Dessa maneira, através da diversidade dos participantes da oficina, foi possível construir uma complexa rede de motivos para a dependência de crack na região. A rede de efeitos provocada por tais forças motrizes está representada na Figura 2. 


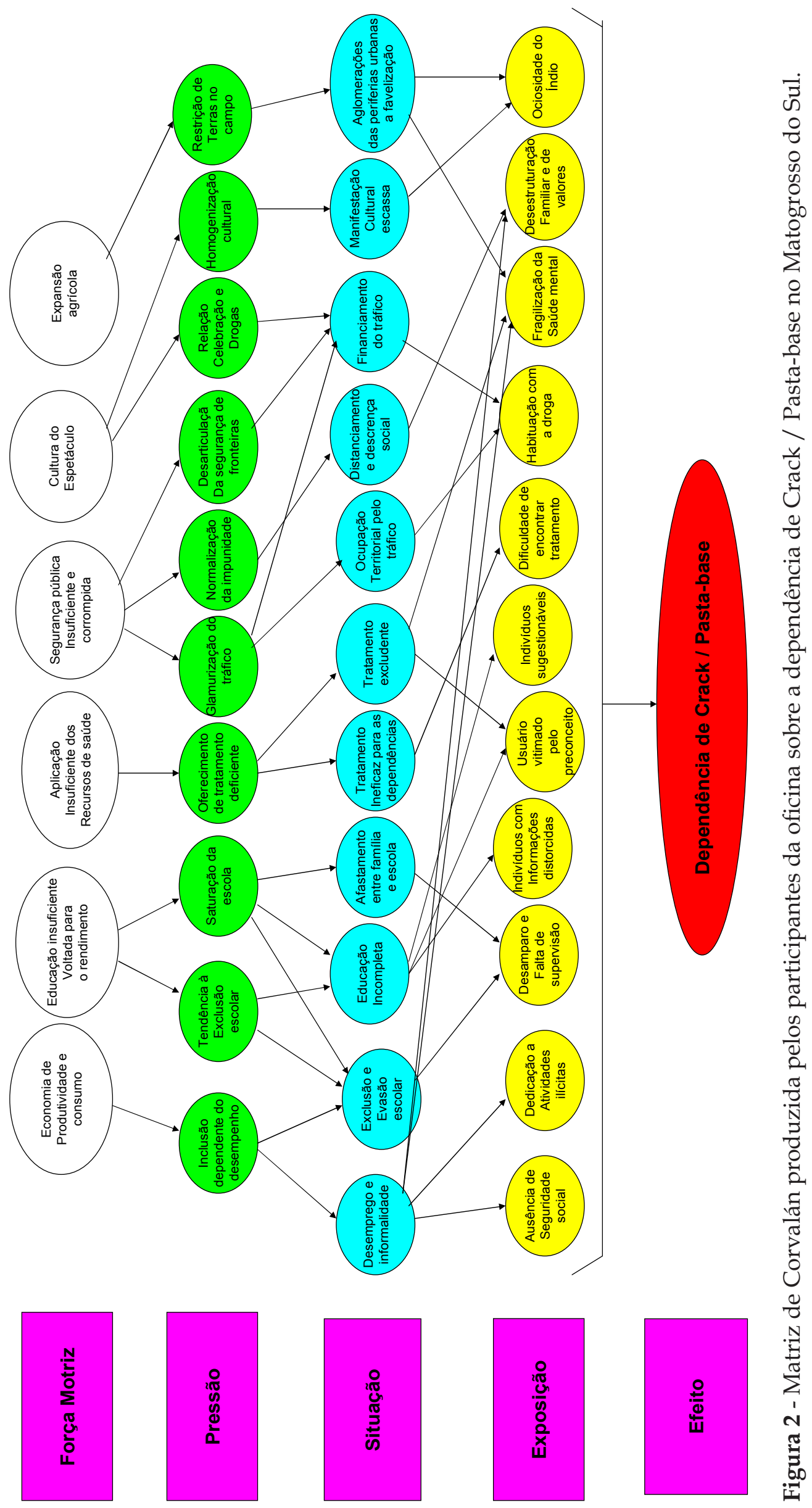

INTERAÇÕES, Campo Grande, MS, v. 17, n. 1, p. 126-133, jan./mar. 2016. 
As relações determinantes para a dependência de crack representadas na figura 2 foram descritas pelos participantes da seguinte forma:

1. Economia-Produtividade-Agricultura: “Em uma economia baseada na produtividade e no consumo, uma porção dos indivíduos em condições menos favoráveis permanece desempregada, excluída e na informalidade. Simultaneamente, sendo o modelo agrícola também baseado na produtividade, há uma ocupação do campo pelo agronegócio, promovendo uma restrição das terras e migração do pequeno produtor e do índio para centros urbanos, onde ocuparão as áreas periféricas. Tais áreas, não sendo providas de infraestrutura e planejamento adequados, constituem favelas. Seja por uma marginalização territorial, seja por uma marginalização econômico-social, são produzidas ausência de seguridade (insegurança) social com desestruturação familiar, dedicação a atividades ilícitas, convívio com estressores (violência, pobreza, doenças infecto-parasitárias etc.) que provocam fragilização da saúde mental e ociosidade de alguns indivíduos pertencentes a outras culturas, como a indígena. Esse último aspecto é particularmente importante, pois MS tem uma das maiores populações indígenas do país".

2. Política Educacional: "Uma educação com recursos insuficientes gera uma saturação da escola. Ao mesmo tempo, um modelo educacional voltado para o desempenho gera uma tendência à exclusão de parte dos alunos. A saturação da escola e a exclusão de alunos, ainda dentro dela, promovem um afastamento entre família e educadores, uma educação incompleta e evasão em casos mais graves. Por tais situações, a criança ou jovem fica privado da assistência e supervisão institucional, sugestionável pela falta de informações, e com a saúde mental fragilizada por um não desenvolvimento cognitivo pleno. Por outro lado, os demais membros da sociedade se mantêm portadores de informações distorcidas e de preconceitos contra os usuários de drogas, e os valores ético-morais padecem pela ineficácia da educação."

3. Política de Saúde: "Há uma aplicação insuficiente dos recursos da saúde, provocadora de oferta deficiente de tratamento. Cria-se assim uma situação de atenção à saúde excludente e ineficaz, que mantém o usuário em dificuldade de encontrar tratamento, continuamente vitimado pelo preconceito e pela fragilização da saúde mental existentes."

4. Cultura: A "existência de uma "cultura do espetáculo" favorece o uso de drogas por sua relação com a celebração, financiando a indústria do álcool e o tráfico e promovendo uma habituação com a existência de drogas nos ambientes festivos. Em paralelo, a "cultura do espetáculo" também produz uma homogeneização e escassez cultural, deixando algumas populações minoritárias, como a indígena, ociosas."

5. Segurança Pública: “Uma segurança pública insuficiente não consegue se articular para produzir controle das fronteiras com a Bolívia e o Paraguai, permitindo grande entrada de drogas no país através de MS. Em paralelo, a corrupção nas instituições garantidoras da lei provoca um senso de normalização da impunidade e uma glamourização de organizações criminosas e dos traficantes de drogas. Todos esses aspectos provocam um aumento do financiamento do tráfico e uma progressiva ocupação territorial em uma sociedade distanciada dos próprios problemas e descrente das possíveis soluções. Ao longo do tempo, ocorre uma progressiva habituação com a droga e, novamente, uma desestruturação da família e dos valores sociais".

\section{DISCUSSÃO}

A diversidade de fatores causais levantados por indivíduos ligados de diferentes maneiras ao suporte de pessoas com dependência de crack / pasta-base torna evidente a complexidade de tal condição. A força motriz mais destacada pelos participantes, por ter criado com maior frequência exposições para a ocorrência da dependência de crack, foi o aspecto da Educação. Muitos estudos observam a relação entre a baixa escolaridade e o uso de crack, mas nenhum deles se debruça sobre os motivos dessa relação. Entre os estudos epidemiológicos que evidenciam a relação entre crack e menores níveis de escolaridade, podemos citar um estudo populacional norte-americano que avaliou a mudança no perfil do usuário de cocaína entre as décadas de 80 
e 90 (MIECH et al., 2005). Foi após o início do consumo do crack na década de 90 que passou a existir uma relação epidemiológica entre uso de cocaína e menores níveis de escolaridade (MIECH et al., 2005).

Dados brasileiros também evidenciam esta relação, ainda que de forma indireta. Em uma revisão sobre o "Perfil dos usuários de crack no Brasil”" (DUALIB et al., 2008) destaca-se que o surgimento da droga ocorreu entre homens pobres, jovens, desempregados e com baixo nível educacional. Observa-se ainda que a prevalência de uso entre escolares é de 0,5\% (ABRAMOVAY; CASTRO, 2005), enquanto que entre menores em situação de rua, a prevalência de uso recente oscila entre 10 e $20 \%$ (NOTO et al., 2003).

A baixa escolaridade também parece se relacionar com a gravidade da dependência, observando-se um perfil de menor escolaridade significativa entre pacientes dependentes de crack internados quando comparados com dependentes de cocaína inalada (GUINDALINI et al., 2006). Já em um estudo brasileiro entre pacientes no nível secundário de atenção em saúde (ambulatorial), não se verificou significância estatística para a diferença de escolaridade (VARGENS et al., 2011). Para explicar a ligação entre educação e uso de crack evidenciada pela literatura, os participantes descreveram um processo de exclusão ligado ao método pedagógico e precariedade institucional.

Um segundo aspecto destacado pelos participantes foi o Econômico, podendo ser agrupado na tríade Economia-Produtividade-Agricultura. Essa constatação da relação entre menores níveis econômicos, exclusão social e uso de crack também pode ser vista com clareza pelos dados da literatura. Santos Cruz et al. (2013), examinando uma amostra de dependentes de crack da comunidade das cidades do Rio de Janeiro e de Salvador, observaram características como instabilidade de moradia, sustento econômico decorrente de práticas legais ou ilegais, história de detenção no último ano e não utilização de serviços sociais e de saúde.

Sutin et al. (2012) observaram que características de comportamento como organização, disciplina e deliberação são protetores para o uso de drogas, e que tais fatores são menos protetores entre indivíduos com menos recursos financeiros. Outro estudo evidenciou que os usuários de crack de áreas marginais de El Salvador consomem mais frequentemente dentro da própria comunidade do que usuários de áreas centrais em decorrência de fatores como a intensidade da transição da população, a coesão e monitoramento da vizinhança, a normalização do uso de drogas e da venda controlada por grupos criminosos e o grau de presença de serviços de prevenção do HIV (DICKSON-GOMEZ, 2010).

Considerando estas relações, os participantes descreveram processos produtores de marginalização de grupos sociais, incluindo a população indígena. Sobre esse último aspecto, apesar da falta de dados sobre a prevalência do uso de crack entre indígenas, observa-se que essa população apresenta particular prevalência para a dependência de substâncias como o álcool e que esta maior frequência se intensifica quanto maior a proximidade entre a comunidade indígena e a sociedade nacional envolvente (GUIMARÃES; GRUBITS, 2007).

A Segurança Pública também foi destacada pelos participantes, sendo vista como "insuficiente e corrompida". Quanto ao aspecto da insuficiência, destaca-se o relatório de auditoria operacional de 2012 do Tribunal de Contas da União sobre o Sistema Nacional de Políticas Sobre Drogas (BRASIL, 2012). Verifica-se, na conclusão, a insuficiência de pessoal, instalações e material da Polícia Federal e a alta rotatividade de agentes, que se estabelecem em regiões inóspitas como o Pantanal, apenas nos primeiros três anos de trabalho. Observa-se ainda grande lentidão para a realização de leilões para a venda de bens apreendidos do tráfico em decorrência de falta de estrutura e desarticulação entre polícia e justiça. O excesso de tempo decorrido gera uma perda do valor econômico desses bens, diminuindo os recursos que poderiam ser aplicados para o combate à entrada de drogas no país e outras ações de prevenção e tratamento dos usuários (BRASIL, 2012). Quanto à corrupção policial, tais eventos podem ser identificados em um estudo de análise de discurso do Rap paulistano, que predominantemente se refere a dois caminhos para o jovem das periferias urbanas (SILVA; SOARES, 2004). Um voltado para a associação com o tráfico, onde se obtém satisfação através 
do poder financeiro e do consumo de bens e de drogas.

O outro voltado para a valorização das relações familiares e de amizade, em que se deve abdicar das satisfações ligadas ao capital (SILVA; SOARES, 2004). Avalia-se no discurso a presença da polícia com uma ação discriminatória, corrompida e arbitrariamente violenta, exercendo uma função antipedagógica na comunidade (SILVA; SOARES, 2004). As descrições dos participantes da presente pesquisa evidenciam a existência de processos semelhantes nas áreas urbanas periféricas de MS.

No aspecto da assistência à saúde, setor onde trabalhavam a maioria dos participantes, foi destacada uma aplicação insuficiente dos recursos, com oferta deficiente de tratamento. No Estado de MS, até o momento da realização desta pesquisa, ainda não tinham sido implementados alguns dispositivos pertencentes à rede de atenção psicossocial financiados pelo Ministério da Saúde para a assistência ao portador de dependências, como os CAPS-ad-III, o Consultório na Rua e as Unidades de Acolhimento. Essa não aplicação de recursos pela gestão pública em associação com a baixa adesão pelos jovens usuários de crack (PEIXOTO et al., 2010; SANTOS-CRUZ et al., 2013) mantém a maioria dos indivíduos sem assistência adequada.

O último elemento apontado foi o cultural, sendo descrita "a existência de uma "cultura do espetáculo", que favorece o uso de drogas por sua relação com a celebração, financiando a indústria do álcool e o tráfico e promovendo uma habituação com a existência de drogas nos ambientes festivos. Foi tendo em vista a necessidade de produção cultural para valorizar a identidade do portador de transtornos mentais na própria comunidade que a política nacional de saúde mental incluiu entre os dispositivos para a assistência e promoção de saúde os Centros de Convivência. Segundo a Portaria n ${ }^{\circ} 396$ de 7 de julho de 2005, nos Centros de Convivência e Cultura devem "ser oferecidos às pessoas com transtornos mentais espaços de sociabilidade, produção e intervenção na cidade.

Através da construção de espaços de convívio e sustentação das diferenças na comunidade e em variados espaços da cidade (os Centros de Convivência), facilita-se a construção de laços sociais e inclusão da pessoa com transtornos mentais". Observa-se assim a necessidade de implementação desse recurso nas redes de atenção psicossocial do Estado do MS.

\section{CONCLUSÃO}

O uso da Matriz de Corvalán para uma condição de saúde diferente das doenças infecto-parasitárias mostrou-se produtivo. Neste caso em particular, para a ampliação do entendimento dos determinantes ambientais para a dependência de crack. A multiplicidade de fatores apontados por profissionais ligados à atenção à dependência de crack em MS torna evidente a complexidade do tema. Revela que, além de uma ação terapêutica que se utiliza da rede de saúde mental, é preciso também de ações intersetoriais (justiça, educação, cultura e economia) para oferecer à população a tríade da promoção-prevenção-tratamento.

Os profissionais envolvidos na oficina apresentam uma clara impressão dessa necessidade, o que fez com que destacassem mais os aspectos econômico, educacional e jurídico do que as ações da saúde. Apesar disso, além da ampliação do trabalho intersetorial, ainda é preciso implementar muitos recursos de assistência ao usuário de crack na região.

\section{REFERÊNCIAS}

ABRAMOVAY, M.; CASTRO, M. G. Drogas nas escolas - versão resumida. Brasília: Organização das Nações Unidas para a Educação, a Ciência e a Cultura, 2005.

BRASIL. Tribunal de Contas da União/Sistema Nacional de Políticas Públicas sobre Drogas. Relator Ministro Aroldo Cedraz. Brasília: TCU, Secretaria de Fiscalização e Avaliação de Programas de Governo, 2012.

CARLINI, E. A.; GALDURÓZ, J. C.; NOTO, A. R., NAPPO, S. A. I Levantamento domiciliar nacional sobre uso de drogas psicotrópicas no Brasil (2001). São Paulo: Secretaria Nacional Antidrogas/Centro Brasileiro de Informações sobre Drogas Psicotrópicas, 2002.

CARLINI, E. A. (Sup.); GALDURÓZ, J. C. (Coord.) et al. II Levantamento domiciliar sobre o uso de drogas psicotrópicas no Brasil: estudo envolvendo as 108 maiores cidades do país - 2005. São Paulo: Páginas \& Letras, 2007.

CORVALÁN, C. F.; KJELLSTRÖM, T.; SMITH, K. R. Health, Environment and Sustainable Development. Identifying Links and Indicators to Promote Action. Epidemiology, v. 10, n. 5, p. 656-60, 1999.

DICKSON-GOMEZ, J. Structural factors influencing patterns of drug selling and use in the San Salvador 
metropolitan area. Medical Anthropology Quarterly, v. 24, n. 2, p. 157-181, 2010.

DUALIBI, L. B.; RIBEIRO, M.; LARANJEIRA, R. Profile of cocaine and crack users in Brazil. Cad Saúde Pública, v. 24, s. 4, p. 545-57, 2008.

GUIMARÃES, L. A. M.; GRUBITS, S. Alcoolismo e violência em etnias indígenas: Uma visão crítica da situação brasileira. Psicologia \& Sociedade, v. 19, n. 1, p. 45-51, 2007.

GUINDALINI, C.; VALLADA, H.; BREEN, G.; LARANJEIRA R. Concurrent crack and powder cocaine users from Sao Paulo: do they represent a different group? BMC Public Health, v. 6, p. 10, 2006.

INSTITUTO BRASILEIRO DE GEOGRAFIA E ESTATÍSTICA (IBGE). Censo 2010. Disponível em: < http:/ / www.ibge.gov.br/home/>.

MIECH, R. A.; CHILCOAT, H; HARDER, V. S. The increase in the association of education and cocaine use over the 1980s and 1990s: evidence for a 'historical period' effect. Drug Alcohol Depend, v. 79, n. 3, p. 31120, 2005.

NAPPO, S. A.; SANCHEZ, Z. M.; RIBEIRO, L. A. Is there a crack epidemic among students in Brazil?: comments on media and public health issues. Cad Saúde Pública, v. 28, n. 9, p. 1643-9, 2012.

NOTO, A. R. et al. Levantamento nacional sobre uso de drogas entre crianças e adolescentes em situação de rua nas 27 capitais brasileiras - 2003. São Paulo: Secretaria Nacional Antidrogas/Centro Brasileiro de Informações sobre Drogas Psicotrópicas, 2003.
PASSOS, S. R.; CAMACHO, L. A. B. Características da clientela de um centro de tratamento para dependência de drogas. Rev Saúde Pública, v. 32, p. 64-71, 1998.

PEIXOTO, C.; PRADO, C. H. O.; RODRIGUES, C. P.; CHEDA, J. N. D.; MOTA, L. B. T.; VERAS, A. B. Impact of clinical and socio-demographic profiles in treatment adherence of patients attending a day hospital for alcohol and drug abuse. J Bras Psiquiatr, v. 59, n. 4, p. 317-21, 2010.

SANTOS CRUZ et al. Key drug use, health and socioeconomic characteristics of young crack users in two Brazilian cities. Int J Drug Policy, v. 24, n. 5, p. 432-8, 2013.

SILVA, V. G. B.; SOARES, C. B. Messages about drugs from rap: how to survive in the periferia. Ciência \& Saúde Coletiva, v. 9, n. 4, p. 975-85, 2004.

SUTIN, A. R.; EVANS, M. K.; ZONDERMAN, A. B. Personality traits and illicit substances: The moderating role of poverty. Drug Alcohol Depend, v. 131, n. 3, p. 247-51, 2012.

UNITED NATIONS. Agenda 21: the United nations programme of action from Rio. New York: United Nations, 1993.

VARGENS, R. W.; CRUZ, M. S.; SANTOS, M. A. Comparison between crack and other drugs abusers in a specialized outpatient facility of a university hospital. Rev Lat Am Enfermagem, Spec No, p. 804-12, 2011.

WORLD HEALTH ORGANIZATION (WHO). Sustainable Development and Healthy Environments. Environmental health indicators: Framework and methodologies. Geneva, 1999. 